\title{
EnjoyYourLAB: una aplicación móvil (App) de apoyo a la docencia en los laboratorios de ingeniería hidráulica (Fase II)
}

\section{EnjoyYourLAB: a mobile application that supports the lecturing in the hydraulics laboratories (Phase II).}

\author{
Jorge García Morillo, Rafael González Perea², \& Juan Antonio Rodríguez \\ Díaz ${ }^{3}$
}

Fecha de recepción: 15/09/2018; Fecha de revisión: 27/02/2019; Fecha de aceptación: 20/05/2019

Cómo citar este artículo:

García Morillo, J, González Perez, R \& Rodríguez Díaz, JA. (2019). EnjoyYourLAB: una aplicación móvil (App) de apoyo a la docencia en los laboratorios de Ingeniería Hidráulica. Revista de Innovación y Buenas Prácticas Docentes, 8(2), 45-55.

Autor de Correspondencia: jgmorillo@uco.es

\begin{abstract}
Resumen:
EnjoyYourLAB es una aplicación móvil (APP) de apoyo a la docencia de las prácticas de laboratorio de Ingeniería Hidráulica. En esta segunda fase se ha depurado la aplicación y se ha completado con la incorporación de la recreación virtual del laboratorio de la Escuela Politécnica Superior de Belmez y con la edición de los vídeos de las prácticas de laboratorio. Se han editado vídeos docentes de las prácticas de laboratorio referentes a medida de presiones y aforo de caudales, pérdidas de carga en tuberías y singularidades, determinación de la curva característica de una bomba y punto de funcionamiento, régimen uniforme y gradualmente variado en canales abiertos y determinación de las fuerza de empuje sobre superficies planas sumergidas. A través de la App los alumnos tendrán acceso a la plataforma Moodle, a las memorias de prácticas, material audiovisual e información técnica de los distintos dispositivos empleados. A través de esta herramienta los estudiantes tendrán disponible un mayor nivel de información, que podrán consultar y visualizar tantas veces cómo deseen lo que contribuirá a la solidez del conocimiento adquirido.
\end{abstract}

Palabras clave: Aplicación móvil, laboratorios de hidráulica, TIC, virtualización.

\begin{abstract}
:
EnjoyYourLAB is a mobile application that supports the practical lessons in the hydraulics laboratory. In the second phase, the App has been updated with a virtual rendering of the Lab at the Advanced Polytechnic School of Belmez and with the edited videos of the practical sessions. The videos edited are about the pressure and flow measurements, head losses in pressurized pipes, characteristics curves of a pump, steady and variable flow in open channels, and the estimation of the hydrostatic forces in submerged surfaces Also students have access to the moodle platform, guidelines for the practical exercises, audios, and technical information. Through this tool the students have access to a higher level of data, which can check at any time, and the number of time required to improve the robustness of the acquired knowledge.
\end{abstract}

Key Words: mobile App, hydraulics laboratories, virtual teaching, ICT.

\footnotetext{
1 Universidad de Córdoba (España), jgmorillo@uco.es; CÓDIGO ORCID: 0000-0003-3385-5462

2 Universidad de Córdoba (España), g72goper@uco.es; CÓDIGO ORCID: 0000-0001-6169-0787

3 Universidad de Córdoba (España), ma2rodij@uco.es; CÓDIGO ORCID: 0000-0002-9621-7786
} 


\section{INTRODUCCIÓN}

La competencia digital es una de las 8 competencias clave que cualquier joven debe haber desarrollado al finalizar la enseñanza obligatoria para poder incorporarse a la vida adulta de manera satisfactoria y ser capaz de desarrollar un aprendizaje permanente a lo largo de la vida, según las indicaciones del Parlamento Europeo sobre competencias calve para el aprendizaje permanente (Recomendación 2006/962/CE del Parlamento Europeo y del Consejo, de 18 de diciembre de 2006). La competencia digital no sólo proporciona la capacidad de aprovechar la riqueza de las nuevas posibilidades asociadas a las tecnologías digitales y los retos que plantean, resulta cada vez más necesaria para poder participar de forma significativa en la nueva sociedad y economía del conocimiento del siglo XXI (Intef, 2017).

En la Conferencia Mundial sobre la Educación Superior, se expone que los estudiantes deben asumir la responsabilidad de ser un participante activo en la adquisición de conocimientos, valores y habilidades necesarios para aprender a conocer, hacer, trabajar en equipo, a ser solidario, tomar decisiones, resolver problemas, etcétera (UNESCO, 2008). Otra recomendación es la de crear nuevos entornos pedagógicos, que van desde los servicios de educación a distancia hasta el establecimiento de sistemas virtuales de enseñanza superior, capaces de establecer sistemas de educación de alta calidad, favoreciendo el progreso social, económico, la sostenibilidad, así como otras prioridades sociales importantes.

Las transformaciones y avances vertiginosos que en materia de ciencia, tecnología e información estamos viendo están originando un nuevo contexto social en que los ciudadanos cada día deben asumir los cambios y retos que le impone la sociedad de la información y el conocimiento. La expansión de las tecnologías de la comunicación e información ha ampliado sus usos en la última década, gracias a las aplicaciones de la web 2.0 que permite la creación de blogs, wikis y algunos espacios virtuales, con los cuales se puede interactuar de forma más dinámica e innovadora (García Sánchez, M., et al, 2017).

Por tanto, se pone de manifiesto que en un proceso de enseñanza-aprendizaje juegan un papel importante las tecnologías de la información y comunicación como apoyo a la interacción con actividades didácticas que integran lo visual, novedoso e interactivo; incentiva el uso de Aplicaciones (App), plataformas y redes sociales; promueve nuevas formas de enseñanza; facilita la búsqueda de información y comunicación, el desarrollo de actividades prácticas del quehacer docente como las videoconferencias, o la edición de vídeos docentes de calidad que permite a los alumnos tener acceso a conceptos teóricos o prácticos de mayor dificultad en cualquier momento del día y la veces que sean necesarias.

Por este motivo y durante el curso académico 2016-2017 este grupo docente se embarcó en un ambicios proyecto de innovación docente (PID) que tenía como objetivo el desarrollo de una aplicación móvil (EnjoyYourLAB) de apoyo a la docencia de las clases de prácticas que se imparten en los laboratorios de ingeniería hidráulica del Campus de Rabanales y de la Escuela Politécnica Superior de Belmez (EPSB).

Las prácticas de laboratorio, de carácter obligatorio, son superadas mediante la realización y superación de un informe de prácticas. La necesidad de aplicar los conceptos teóricos-prácticos impartidos en el aula a casos reales en el laboratorio y su posterior redacción a través del informe de prácticas genera serias dudas a los alumnos, demandando en ocasiones un elevado número de horas de tutoría o incluso la repetición de las prácticas con otros grupos. 
Además, esta situación se acentúa en aquellos alumnos que por alguna razón justificada no han podido asistir a dichas prácticas. Por dicho motivo, se ha querido dotar al alumno de una herramienta interactiva que, de una forma directa y fácil, tenga acceso a información útil de los distintos dispositivos hidráulicos empleados en las prácticas, de las memorias e informes a realizar, así como de material audiovisual (presentaciones, imágenes, vídeos) editado por el equipo docente, o desarrollado por otras Escuelas y disponibles en la red.

Debido a la robustez y versatilidad con la que se ha diseñado, EnjoyYourLab se podría implementar, con ajustes mínimos, en cualquier laboratorio de enseñanzas técnicas. Con este PID se van a beneficiar numerosas asignaturas del área de conocimiento de Ingeniería Hidráulica de los Grados de Ingeniería Agroalimentaria y del Medio Rural e Ingeniería Forestal de la Escuela Técnica Superior de Ingenieros Agrónomos y de Montes (ETSIAM), y de los grados de Ingeniería Civil e Ingeniería en Recursos Energéticos y Mineros pertenecientes a la EPSB.

El objetivo general que se planteó cuando iniciamos este PID hace dos cursos fue el de motivar e incentivar al alumno al estudio de la Ingeniería Hidráulica como disciplina fundamental de los Grados mencionados, mediante la creación de una herramienta interactiva (App) que mejorara sustancialmente la metodología de transferencia del conocimiento teórico al práctico. En una primera fase se desarrolló inicialmente la App y se hizo una recreación virtual del laboratorio de hidráulica del Campus de Rabanales.

En esta segunda fase, el objetivo principal ha sido depurar la App EnjoyYourLAB y darle contenido para que esté operativa y se pueda utilizar durante el curso 20182019. Para ello se planteó llevar a cabo las siguientes tareas antes de lanzar la aplicación y que estuviera disponible para los alumnos en Play Store:

- Darle contenido al laboratorio virtual de Rabanales. Subir las memorias e informes de prácticas, cargar información técnica y útil de los distintos dispositivos del laboratorio que los alumnos se podrán descargar.

- Recrear virtualmente el laboratorio de hidráulica de Belmez e incorporarlo a la APP. Dotarlo con las memorias e informes de prácticas así como información útil de los dispositivos hidráulicos empleados en las prácticas.

- Editar las prácticas de laboratorio en vídeo con calidad profesional con la ayuda del servicio de Ucodigital. Esto permitiría a los alumnos poder ver las prácticas tantas veces como deseen para afianzar los conceptos teóricos, el procedimiento, la toma de datos y los cálculos a desarrollar.

\section{DESARROLLO DE LA EXPERIENCIA DE INNOVACIÓN}

Durante el curso 2016-2017 se ha diseñado y desarrollado EnjoyYourLab, una App dinámica, versátil y robusta para dispositivos móviles con sistema operativo Android. Esta App se podrá instalar de forma fácil y gratuita por los alumnos de la asignatura desde el repositorio oficial de Android (Play Store). La App para dispositivos Android ha sido desarrollada con el software de desarrollo Android Studio, versión 3.2.2. y se ha hecho la recreación virtual del laboratorio de Hidráulica del Campus de Rabanales mediante el software de diseño SketchUp, versión 2017, dotándola de un contenido mínimo para poder ser testada. 


\subsection{Laboratorio virtual de Ingeniería Hidráulica del Campus de Rabanales}

EnjoyYourLab está estructurada en 3 secciones: Laboratorio, Asignaturas y Moodle. La primera sección denominada Laboratorio contiene una recreación virtual y dinámica del laboratorio de hidráulica del Campus de Rabanales (figura 1, figura 2, figura 3).
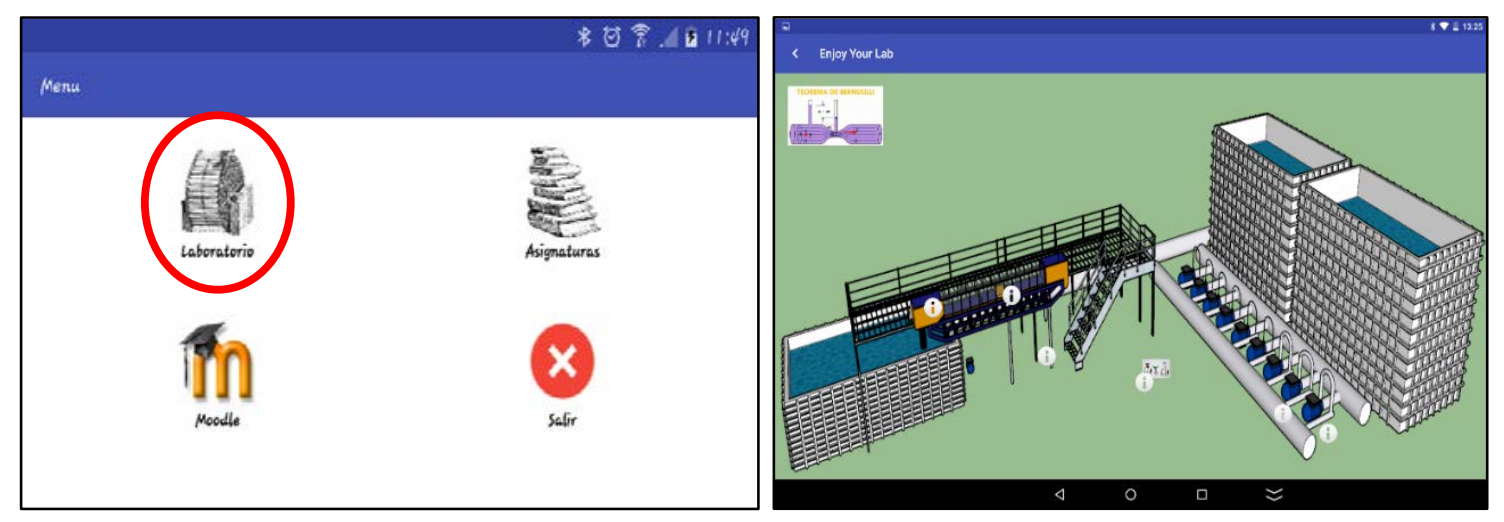

Figura 1. Estructura de la App con acceso a Laboratorios, Asignaturas y Moodle.
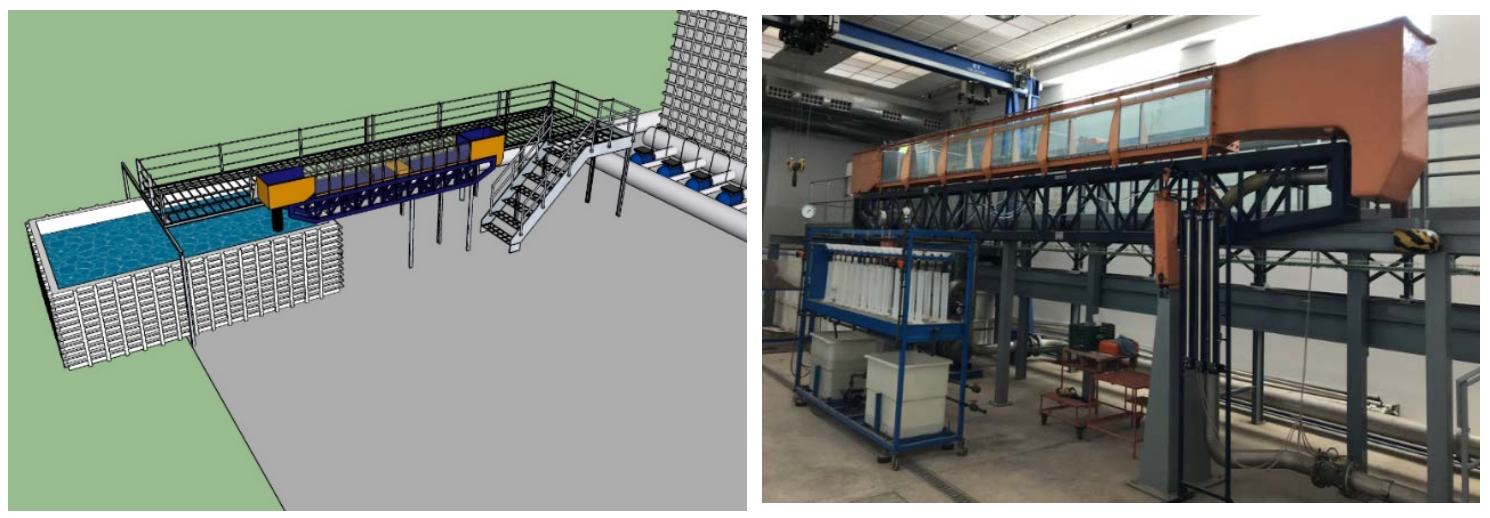

Figura 2. Imagen virtual del laboratorio de hidráulica del campus de Rabanales.
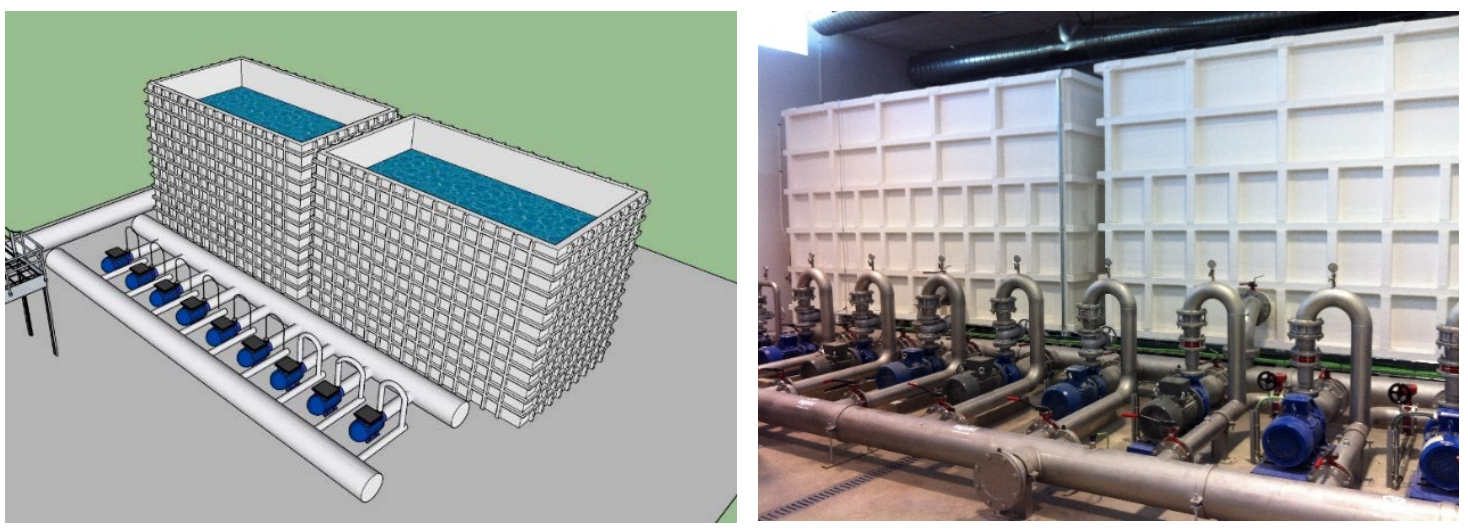

Figura 3. Imagen virtual del laboratorio de hidráulica del campus de Rabanales. 
En esta segunda fase se ha incorporado información de los equipos utilizados, la memoria de prácticas y los vídeos docentes de cada una de las prácticas. Así, seleccionando cada uno de los equipos se abrirá una descripción teórica de los mismos, así como un video explicativo del desarrollo de la práctica y la memoria de la misma con el fin de que el alumno pueda recrear fuera del horario de prácticas el trabajo de ese equipo, visto en el laboratorio (figura 4, figura 5).
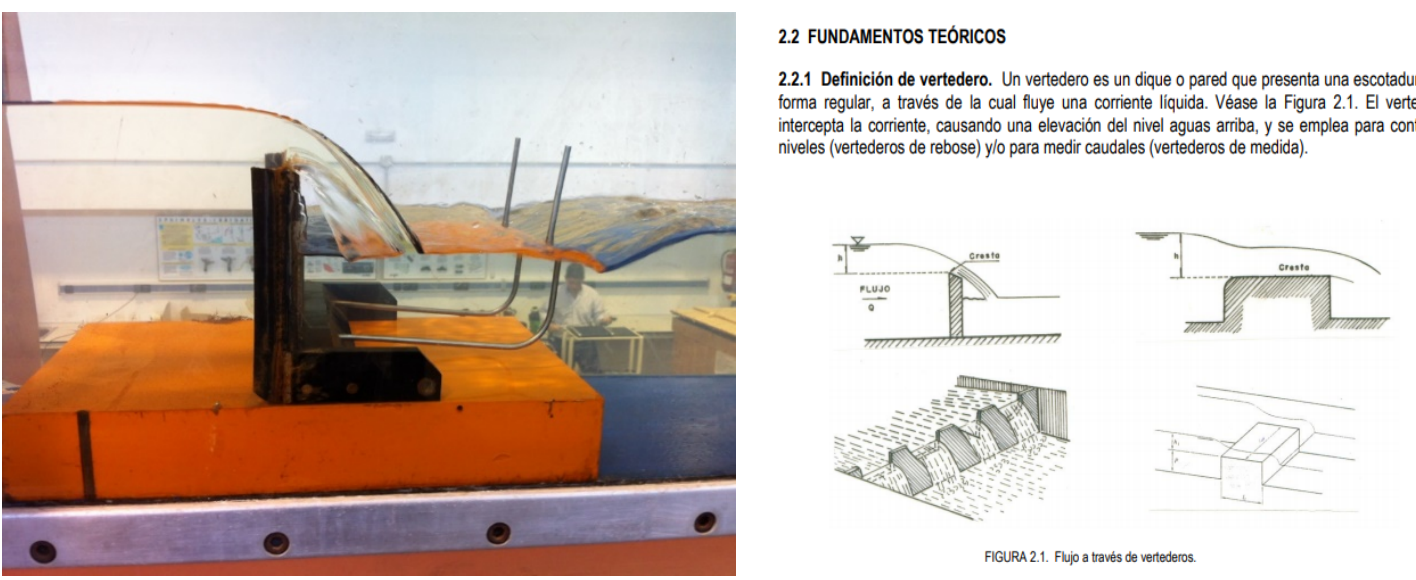

Figura 4. Imagen con información sobre resalto hidráulico subido a la App.

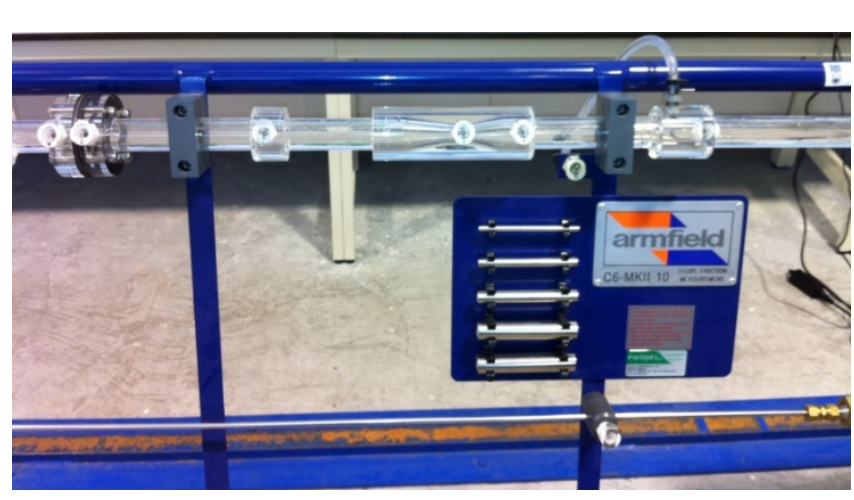

Posibilidades Didácticas

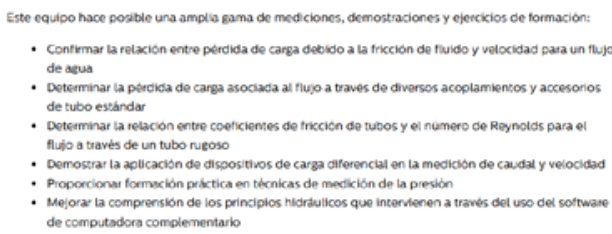

Descripción

La tricción en los tubos es uno de los experimentios clasicos de laboratorio y siempre ha tenisio un lugar en la enserenanza pactica de la mecinica de fluidor

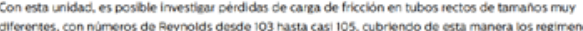

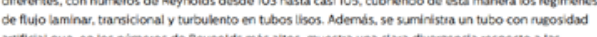

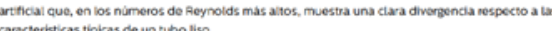

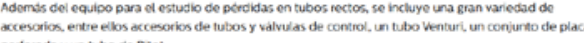

Figura 5. Información técnica subida a la App sobre el banco de ensayos.

La segunda sección de la App denominada Asignaturas contiene información sobre las asignaturas del Área de Ingeniería Hidráulica que tienen sesiones de prácticas en el laboratorio, así como, docentes, cursos en las que se imparten y cuatrimestre (Fig. 6). Seleccionando cada una de estas asignaturas, el alumno puede acceder a los documentos relacionados con cada una de las practicas desarrolladas (informe de prácticas, material adicional, etc.). Tanto la información del laboratorio virtual como la de las asignaturas está almacenada en un repositorio de la Universidad de Córdoba y es accesible y modificable por el personal docente sin tener que modificar la App. Así, EnjoyYourLab está dotada de un gran dinamismo y versatilidad. 
Finalmente, la última sección de la App, Moodle, cuenta con un enlace directo a la plataforma Moodle, de tal manera que el alumno pueda acceder de una forma dinámica a dicha plataforma. Así, a través de la App y el laboratorio virtual, el alumno puede incorporarse a las distintas prácticas que se desarrollan a lo largo del curso, acceder a información adicional en distintos formatos e incluso establecer debates, dudas, etc., tanto con el profesorado como el resto de los compañeros a través del foro de la plataforma Moodle (figura 6).

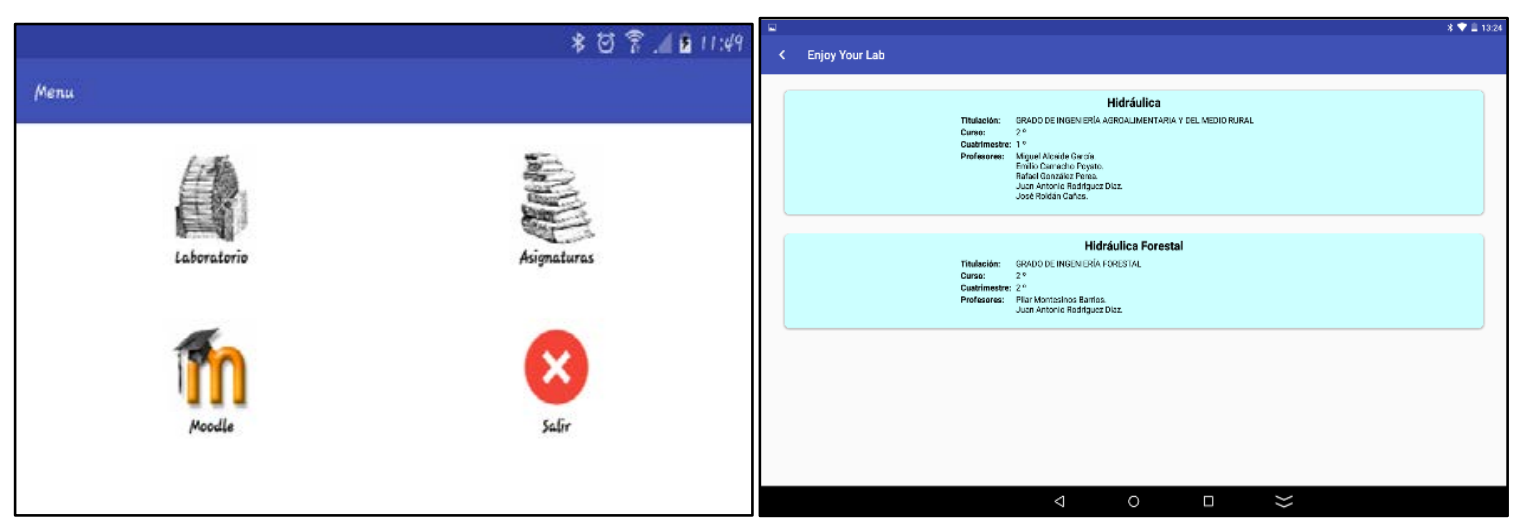

Figura 6. Estructura de la App con acceso a Laboratorios, Asignaturas y Moodle.

En la primera fase del PID se adquirieron dos tabletas, una para cada Laboratorio de Hidráulica, ya que no se pueden compartir debido a que un laboratorio se encuentra en el Campus de Rabanales y el otro en la EPSB. Con las tabletas se pretende que haya en todo momento un dispositivo con acceso a la App en cada laboratorio, que servirá al docente para explicar la herramienta diseñada, así como para que los alumnos puedan interactuar con la misma. Durante el curso próximo, al inicio de las prácticas de laboratorio se dedicará parte de una clase a hacer una introducción a la App, ayudando a los alumnos a descargarla en sus dispositivos móviles, y explicándoles detenidamente el funcionamiento y la estructura de ésta.

\subsection{Laboratorio virtual de la EPSB}

En esta segunda fase se ha completado la aplicación móvil con la recreación virtual del laboratorio de la EPSB (figura 7, figura 8).

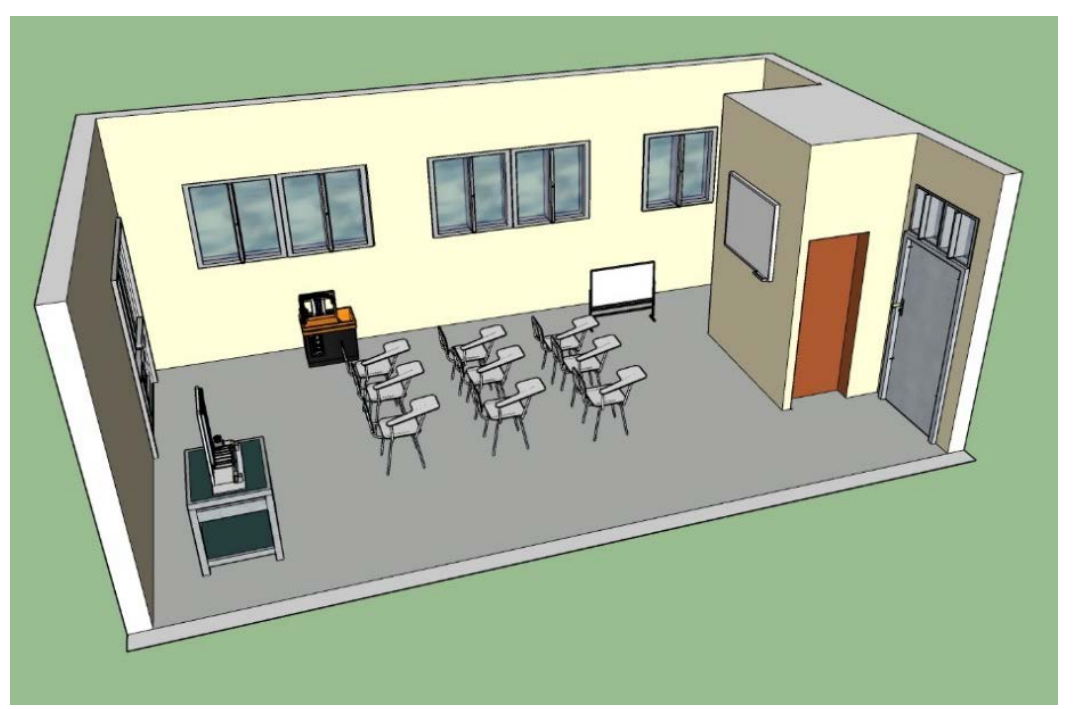

Figura 7. Imagen del laboratorio virtual de hidráulica de la EPSB. 

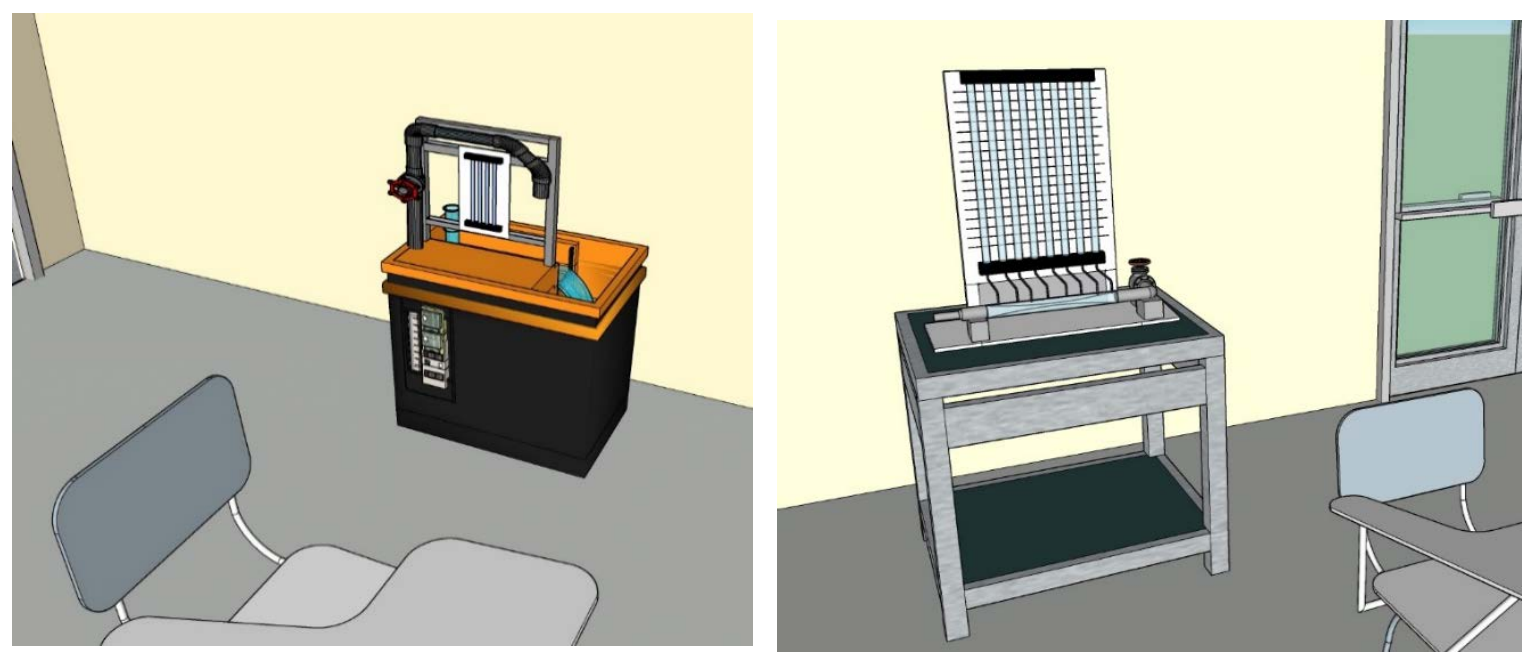

Figura 8. Imagen de detalle de dispositivos hidráulicos del laboratorio de la EPSB.

En el laboratorio virtual, se ha incorporado información útil sobre los distintos dispositivos hidráulicos a utilizar en las prácticas (figura 9), así como la memoria de prácticas que tienen que realizar y la hoja para la toma de datos en laboratorio.

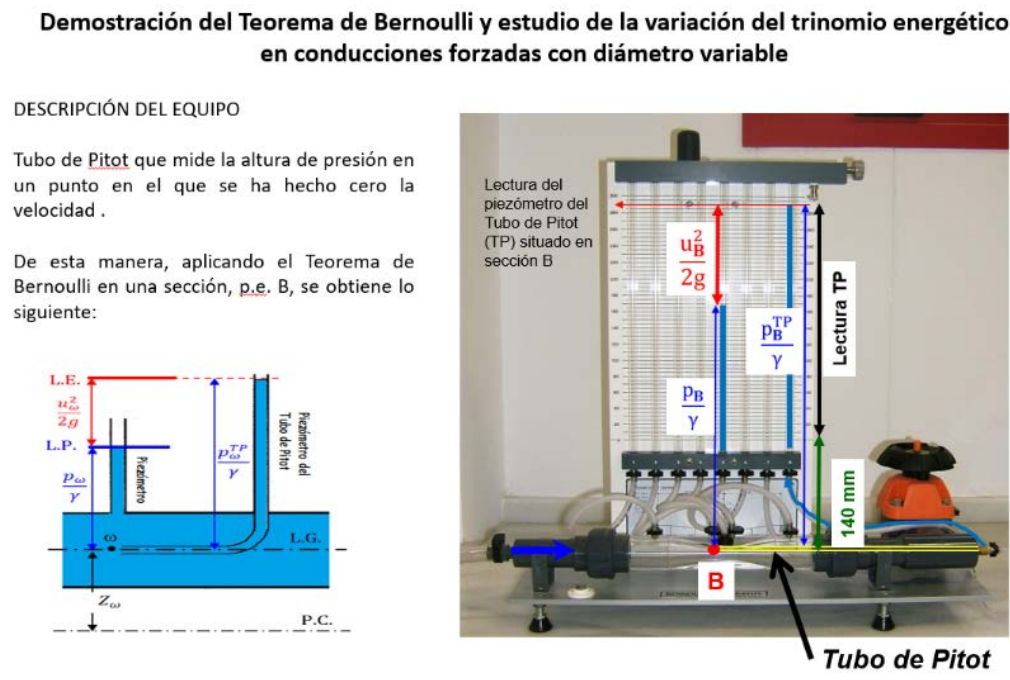

Figura 9. Información sobre dispositivo hidráulico empleado para demostrar el teorema de Bernoulli.

\subsection{Edición de las prácticas de laboratorio en vídeo}

Se han editado en vídeo con calidad profesional cinco prácticas de laboratorio que se realizan en los cuatro grados mencionados al inicio de esta memoria. Aunque la grabación se ha realizado, los vídeos están en fase de edición y estarán disponible para el curso próximo. 
Se han grabado las siguientes prácticas:

- Medida de presiones y aforo de caudales

- Pérdida de carga en tuberías y singularidades

- Determinación de la curva característica de una bomba y punto de funcionamiento

- Régimen uniforme y gradualmente variado en canales abiertos

- Determinación de la fuerza de empuje en superficies sobre superficies planas sumergidas

Cada una de las prácticas grabadas ha seguido el siguiente guion:

Se empieza con una breve introducción de la práctica (figura 10) para a continuación indicar cuáles son los objetivos de la misma.

Corrientes Libres

1. Introducción
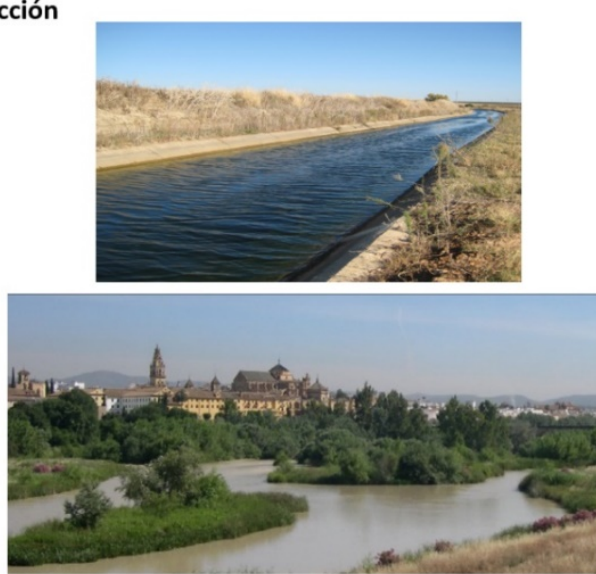

Pérdidas de carga en tuberías y singularidades

1. Introducción
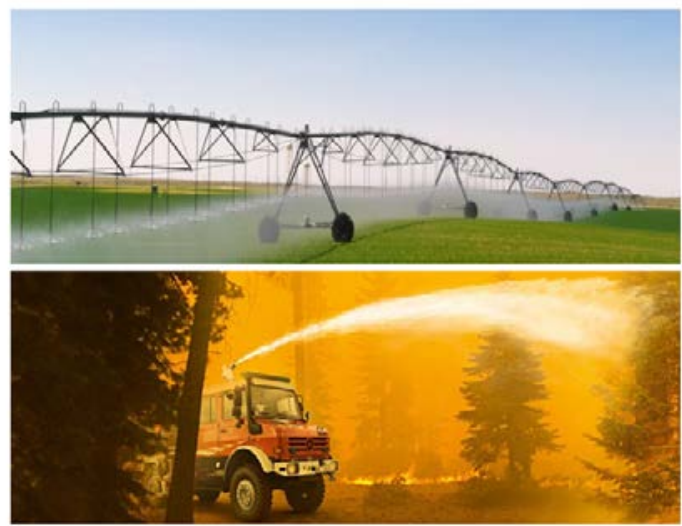

Figura 10. Introducción a las prácticas de corrientes libres y de pérdida de cargas en tuberías.

Posteriormente se explican los equipos y dispositivos de medida que se emplean en la práctica. En la figura 11 se aprecian los equipos e instrumentos de medida y una breve explicación sobre el venturímetro y la vertedero de cresta ancha.

3. Equipo
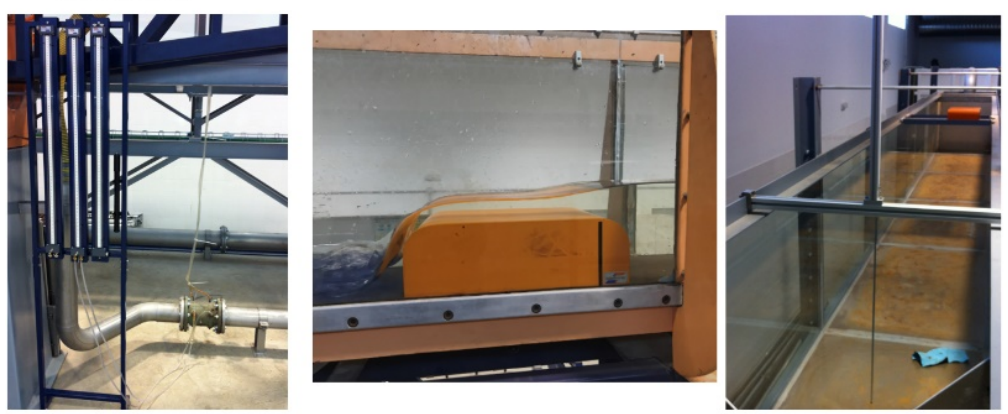

$\checkmark$ Canal de pendiente variable alimentado por una tubería de impulsión.

$\checkmark$ Venturímetro que servirá para mediar el gasto $\mathrm{Q}$.

$\checkmark$ Transición interpuesta en el canal, Vertedero de cresta ancha.

$\checkmark$ Limnímetro para medir el calado. 

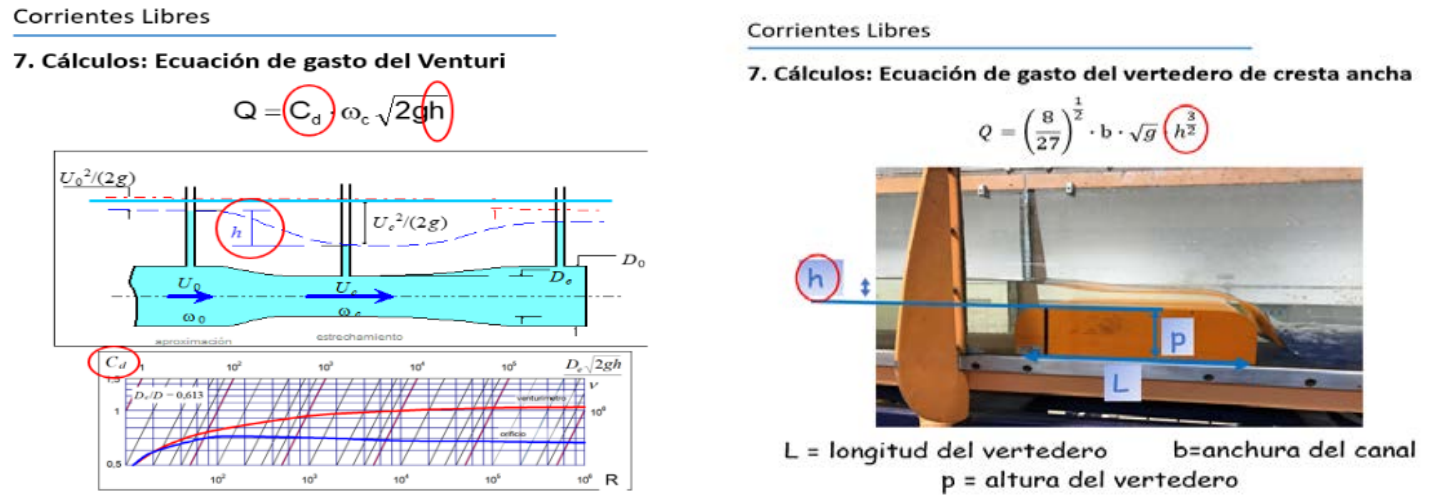

Figura 11. Descripción de los dispositivos a emplear en la práctica de corrientes libres.

Una vez explicados los equipos y dispositivos se hace un recorrido por el procedimiento que se va a realizar en el laboratorio. La figura 12 muestra el procedimiento que se va a llevar a cabo para el desarrollo de las prácticas sobre medidas de pérdidas de carga en conducciones y del cálculo de las fuerzas de empuje en superficies planas sumergidas.
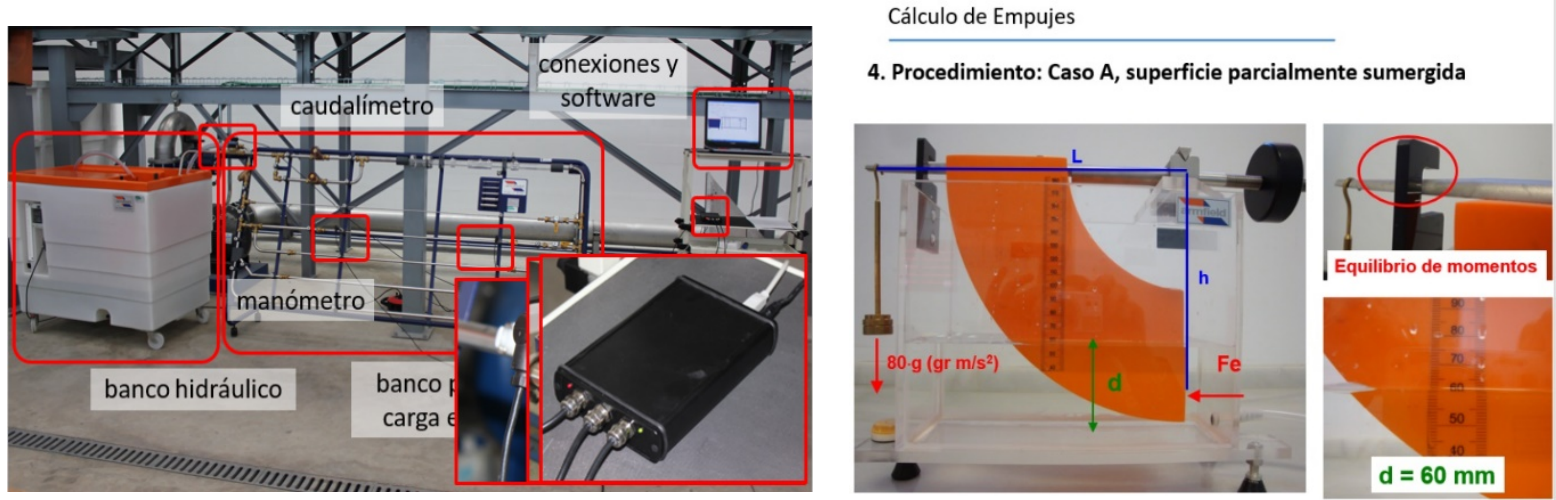

Figura 12. Procedimiento a seguir en laboratorio para el desarrollo de la práctica de corrientes en carga y de empujes.

Finalmente se presenta el informe que tienen que desarrollar, junto a los datos que tienen que medir en el laboratorio (figura 13) y los cálculos y ecuaciones más importantes que tienen que aplicar para realizar dicho informe. 
Pérdidas de carga en tuberias y singularidades

4. Toma de datos en laboratorio

Medidas de altura de presión y caudal
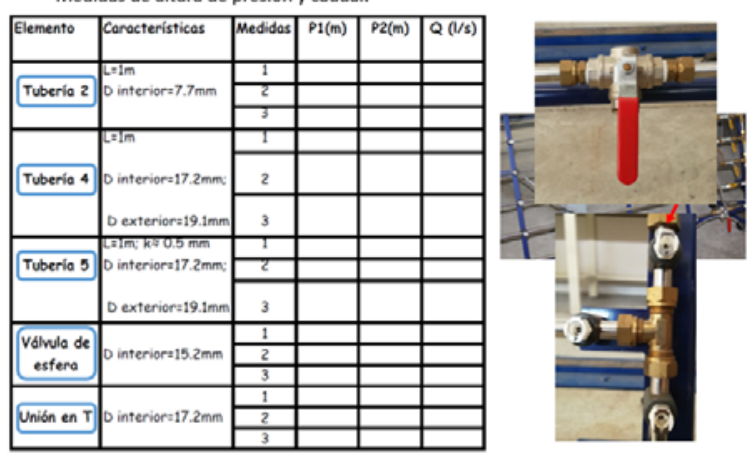

Cálculo de Empujes

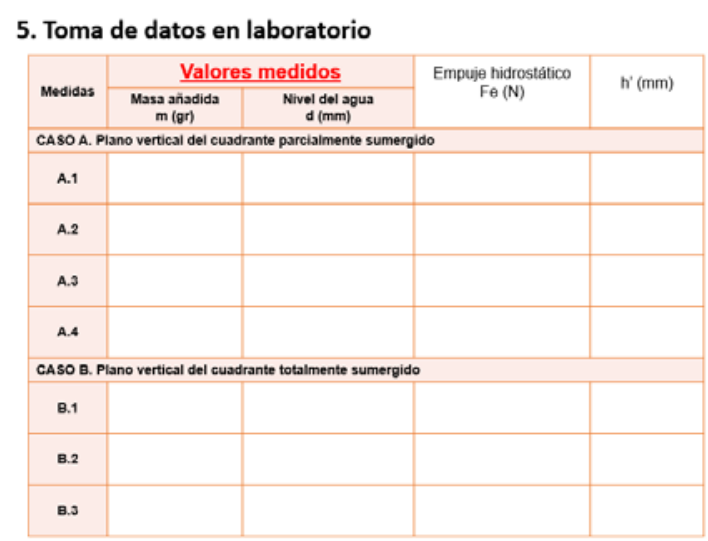

Figura 13. Hojas de toma de datos en laboratorio para la práctica de corrientes en carga y cálculo de las fuerzas de empuje.

\section{RESULTADOS}

Los resultados de este PID son de dos tipos. Por una parte el desarrollo y puesta a punto de la aplicación y edición de los vídeos docentes y por otro lado resultados derivados de la utilización de este herramienta por parte del alumnado.

La App está desarrollada y depurada para su utilización el próximo curso. La App tiene información relativa a los distintos dispositivos hidráulicos, memoria de prácticas y otra información o enlaces de interés para los alumnos. Los vídeos docentes se han grabado y están en fase de edición para su utilización el próximo curso académico. Una vez finalizados los vídeos se subirán a la aplicación móvil para que los alumnos puedan ver el desarrollo de las prácticas las veces que lo deseen. Los vídeos están compuestos de una parte de introducción que se ha grabado en las instalaciones de Ucodigital y otra parte con el desarrollo y toma de datos que se ha grabado en el laboratorio de Ingeniería Hidráulica del Campus de Rabanales.

Los resultados sobre la utilización de la App y sus contenidos como los vídeos docentes todavía no se han podido evaluar. Poner a punto la App, darle contenido y la grabación de las prácticas han supuesto una gran cantidad de trabajo lo que nos ha obligado a posponer su uso para el curso próximo. Durante el curso 2019-2020 se utilizaran la App y los vídeos como herramientas de apoyo a la docencia de las prácticas de laboratorio de hidráulica. Al final de las prácticas los alumnos evaluarán la utilidad de las mismas así como posibles mejoras. 


\section{CONCLUSIONES}

EnjoyYourLAB es una aplicación móvil de utilidad para la docencia práctica en los laboratorios de hidráulica. Esta App permite la incorporación de nuevas herramientas TIC en el proceso de enseñanza-aprendizaje en ingeniería, puede ser de gran utilidad, y está depurada y lista para su uso durante el próximo curso académico.

Esta herramienta nos permite avanzar en la implantación de criterios de enseñanza virtual o semipresencial. En este caso es muy importante, puesto que el laboratorio de la EPSB no dispone de todos los instrumentos o dispositivos que existen en laboratorio del Campus de Rabanales, pudiendo en este caso los alumnos de la EPSB beneficiarse de la instrumentación del laboratorio de Rabanales, de forma virtual y mediante los vídeos docentes que se han grabado con calidad profesional con la ayuda de Ucodigital. De igual modo se podrán beneficiar los alumnos que estén matriculados a tiempo parcial o que por motivos de fuerza mayor no puedan asistir a alguna práctica.

Una vez operativa, lo más importante será comprobar la utilidad y aceptación por parte del alumnado. Esto se llevará a cabo durante las prácticas de laboratorio que se desarrollen en el curso 2019-2020, en el que los alumnos tendrán que descargarla y utilizarla. Al final de las prácticas se analizará la aceptación de la App por parte del alumnado y se les pedirá sus opiniones y sugerencias para la mejora de la misma.

\section{REFERENCIAS}

García Sánchez M. R., Reyes Añorve, J. \& Godínez Alarcón, G. 2017. Las Tic en la Educación superior, innovaciones y retos. Revista Iberoamericana de las Ciencias Sociales y Humanísticas, 6(12).

Intef (2017). Marco Común de Competencia Digital Docente. Instituto Nacional de Tecnologías Educativas y de Formación del Profesorado. Ministerio de Educación, Cultura y Deporte, Gobierno de España.

Recomendación 2006/962/CE del Parlamento Europeo y del Consejo, de 18 de diciembre de 2006, sobre las competencias clave para el aprendizaje permanente [Diario Oficial L 394 de 30.12.2006]. Recuperado de http://europa.eu/legislation_summaries/education_training_youth/lifelong_learni ng/c 11090_e s.htm

UNESCO (2008). Estándares de competencia en TIC para docentes. Recuperado de: http://www.eduteka.org/pdfdir/UNESCOEstandaresDocentes.pdf 